\title{
THE METHOD FOR THE OPTIMAL EXPERIMENT DESIGN
}

\author{
Natalija POZNIAK $\mathbb{B}^{1}$, Leonidas SAKALAUSKAS $\mathbb{D}^{2 *}$ \\ ${ }^{I}$ University of Applied Sciences/Vilniaus Kolegija, Saltoniškiu str. 58, 08105, Vilnius, Lithuania \\ ${ }^{2}$ Vilnius Gediminas Technical University, Department of Information Technologies, \\ Sauletekio str. 11, 10221, Vilnius, Lithuania \\ *E-mail: Leonidas.Sakalauskas@vgtu.lt
}

\begin{abstract}
Purpose - is to develop the Bayesian method of optimal engineering design by a series of experiments, aiming to manage experimental resources in a rational economic way.

Research methodology - is based on modelling of experimental data by Gaussian random fields (GRF) and using matrices of fractional Euclidean distances. Next, the P-algorithm for the planning of the experiment series is created in order to optimize the values of the response surface.

Findings - the application of the developed method in engineering design enable us to create plans for the experiment series in order to create new functional products and processes managing experimental resources in a rational economic way.

Research limitations - the creation of the plans of the experiment series can require a large amount of computer time related to the application of the Monte Carlo procedure in order to ensure the optimality of created plans. However, this limitation can be avoided using distributed computing tools.

Practical implications - The created method helps engineers to seek solutions to experimental problems, considering the economic viability of each potential solution along with the technical aspects.

Originality/Value - in creating functional products and processes engineers are using the experimental design process, which usually is highly iterative. The developed approach enables us to design the experimental series inflexible way, decreasing the number of required experiments and avoiding of rather expensive methods such as factorial experiments, steepest descent, etc., usually applied for experimental design in engineering practice.
\end{abstract}

Keywords: Design of experiments, Bayesian method, Gaussian random fields, the economics of engineering, response surface.

\section{JEL Classification: C14}

Conference topic: Contemporary Issues in Business, Management and Economics Engineering.

\section{Introduction}

Application of economic principles in engineering is related to decision making regarding the allocation of limited resources (Weinberger, Sha, Saul, 2004; Morris, 2013). Engineers seek solutions to problems, and the economic viability of each potential solution is normally considered along with the technical aspects. In creating functional products and processes engineers are using the experimental design process, which usually is highly iterative. Since parts of design process often need to be repeated many times the problems arise with resource allocation exploring sets of possible alternatives (Box \& Draper, 2007; Bijan \& Gorjidooz, 2016; Carpio, Giordano, \& Secchi, 2017). Thus, engineering design problems often require to create experiment series in order to optimize the values of the response surface that depends on various variables. This paper deals with the Bayesian experiment design method, based on the modelling of observation data obtained during the experiments by Gaussian random fields (GRF). That allows us to include the predictable information about the design variables into the model to be identified and to estimate the uncertainties of the observations. The novelty of the developed model is the application of matrices of fractional Euclidean distances between experimental observations points to model the covariances of the GRF. Thus, such a model enables us to avoid the application of positive correlation functions usually applied for creation of the GRF. Using the proposed model the P-algorithm for the planning of the experiment series is created in order to optimize the values of the response surface that depends on various variables. The algorithm is maximizing the expected increment in the response surface, 
depending on measured experimental data, which is a complex multivariate integral in general. For evaluation of this integral and maximization of the expected increment the Monte Carlo method is developed in the Sampling Average Approximation framework. The created method is investigated by computer simulation for solving benchmark examples and is applied to the engineering problem: optimal design of wastewater filters. Hence, the developed approach enables us to design the experimental series in a flexible way, decreasing the number of required experiments and avoiding of known but rather expensive methods (Nooshin \& Hamid, 2017; Maged, Haridy, Shamsuzzaman, Alsyouf, $\&$ Zaied, 2018). Although planning of series requires rather a long Monte Carlo computer simulations time the created method presents an economically and managerially efficient tool for the design of new engineering objects by a series of experiments.

\section{Modelling of experimental data by a Random Gaussian field}

Design of experiments is a systematic approach to engineering problem aiming at predicting the outcome by introducing a change of the preconditions, which is represented by one or more independent variables, also referred to as "input variables" or "predictor variables." The change in one or more independent variables is generally hypothesized to result in a change in one or more dependent variables, also referred to as "response variables” or values on „response surface“ or „response function“. Experimental design involves not only the selection of suitable independent, dependent, and control variables but planning the experiments in an optimal way under given the constraints of available resources. There are multiple approaches for determining the set of design points (unique combinations of the settings of the independent variables) to be used in the experiment. Note that, recently engineers are using an experimental design practice such traditional technologies as factorial experiments, steepest descent, Box-Wilson procedure, etc., which usually are economically and managerially inefficient, because require to perform a large number of experiments at customizable established levels, etc. (Box \& Draper, 2007; Nooshin \& Hamid, 2017; Maged et al., 2017). The statistical model also applied in a specific knowledge-based system to assist designers in configuring numerical design of experiments processes efficiently by taking into account variabilities and uncertainties (El-Gamal \& Palfrey, 1996; Carpio et al., 2017; Blondet, Le Duigou, \& Boudaoud, 2019).

In this paper, the probabilistic model of response surface is considered, which values are obtained by observation, physical measurements or computer simulation, etc. Due to the fact that there are no further data except the measurement performed at the experimental points, the surface which represents the objective response function can be designed as a homogeneous Gaussian random field (GRF) $Z(x, \omega)$, which is a measurable function of random event $\omega$ in some probability space (Jones, 2001). Let us consider GRF with the constant mean vector:

$$
E Z(x, \omega)=\mu \cdot E,
$$

and the covariance matrix

$$
E(Z(x, \omega)-\mu \cdot E) \cdot(Z(x, \omega)-\mu \cdot E)^{T}=\beta^{2} \cdot F,
$$

where $\mu$ and $\beta$ are parameters, $\beta>0, E$ denotes a $K$-dimensional vector-column of units, and $F$ is a positively defined matrix, the choice of which is discussed below.

In this paper, a data model is developed, using the distances with fractional Euclidean distance matrices (Pozniak \& Sakalauskas, 2017; 2019). Since it is unknown which of all the function variables are preponderant, they are considered as equivalent, and, thus, fractional Euclidean distances between the measurement points are calculated, that now are symmetric with respect to miscellaneous variables. Let a data set $\tilde{X}=(X, \hat{X})$ be given, that contains suba set

$$
X=\left(x_{1}, x_{2}, \ldots, x_{N}\right)
$$

consisting of $N$ vectors, where experiments_have been done and surface values obtained after physical experiments or computer simulations, etc., as well as the respective subset

$$
\hat{X}=\left(x_{N+1}, x_{N+2}, \ldots, x_{K}\right),
$$

consisting of vectors to be planned for a series of new experiments, where $x_{i} \in \mathfrak{R}^{d}, 1 \leq i \leq K, d \geq 1$. Let us denote a set of values of the known response function values as

$$
Y=\left(y_{1}, y_{2}, \ldots, y_{N}\right)^{T},
$$

$0<N<K$, that were calculated for the set of points $X$. 
In order to create the GRF model, the extended set $\left(\tilde{X}, x_{c}\right)$ is considered (Pozniak\&Sakalauskas (2017), Pozniak\&Sakalauskas (2019)). Let us denote a $(K+1) \times(K+1)$ matrix

$$
\tilde{A}=\left[\left(\left|x_{i}-x_{j}\right|\right)^{\delta}\right]_{i, j=1}^{K},
$$

degrees $\delta$ of Euclidean square distances between the vectors of sthe et $\left(\tilde{X}, x_{c}\right)$, where $0 \leq \delta \leq 1$, $\left|x_{i}-x_{j}\right|=\left(x_{i}-x_{j}\right)^{T} \cdot\left(x_{i}-x_{j}\right), 1 \leq i \leq K$. Note that $\delta=\frac{1}{2}$ in a special case of the usual Euclidean distances. Usually, the main tool for $t$,,e study of Euclidean distance matrix is the kernel matrix used for modelling of GRF covariates (Weinberger (2004), Pozniak and Sakalauskas (2017). It is proved in (Pozniak \& Sakalauskas, 2017, 2019), that this matrix is positively defined, therefore such a choice is correct.

Thus, the statistical model of the experimental data is presented in a simple and clear for designers way using only the matrix of pairwise distance between experimental points.

\section{Estimation and prediction of a Gaussian random field}

Let us consider an approach for the experimental data prediction based on modelling by GRF. Assume the data be modelled by the probabilistic Gaussian model with a constant mean (1) and the covariance matrix of type (2). Now let us decompose the matrix of fractional Euclidean square distances between points of $\tilde{X}$ :

$$
\tilde{A}=\left[\begin{array}{ll}
A & a \\
a^{T} & \eta
\end{array}\right]
$$

where $A$ is a submatrix of fractional degrees of Euclidean square distances between points of set $X, a$ is a submatrix of fractional degrees of Euclidean square distances between points of $X$ and $\hat{X}, \eta$ is a submatrix of fractional degrees of Euclidean square distances between points of $\hat{X}$.

Specifically, the prediction of the response function in a subset $\hat{X}$ is obtained as an a posteriori mean, using the vector of known values $Y=\left(y_{1}, y_{2}, \ldots, y_{N}\right)^{T}$, got by experimenting with subset $X$. Denote the values of GRF in a subset $\widehat{X}$ by

$$
\hat{Y}=\left(y_{N+1}, y_{N+2}, \ldots, y_{K}\right)^{T}
$$

$y_{i}=Z\left(x_{i}, \omega\right), N<i \leq K$. Using the expressions of the a posteriori mean vector and the a posteriori covariance matrix (Pozniak \& Sakalauskas, 2017, 2019), one can get from:

$$
\begin{gathered}
y(\hat{X})=E(\hat{Y} \mid Y)=Y^{T} \cdot A^{-1} \cdot\left(a+E \cdot \frac{\left(\varepsilon^{T}-E^{T} \cdot A^{-1} \cdot a\right)}{E^{T} \cdot A^{-1} \cdot E}\right) \\
\beta(\hat{X})=E\left((\hat{Y}-E(\hat{Y} \mid Y)) \cdot(\hat{Y}-E(\hat{Y} \mid Y))^{T} \mid Y\right)= \\
\hat{\beta}^{2} \cdot\left(a^{T} \cdot A^{-1} \cdot a-\eta-\frac{\left(\varepsilon-a^{T} \cdot A^{-1} \cdot E\right) \cdot\left(\varepsilon^{T}-E^{T} \cdot A^{-1} \cdot a\right)}{E^{T} \cdot A^{-1} \cdot E}\right),
\end{gathered}
$$

and

$$
\hat{\beta}^{2}=\frac{1}{K} \cdot\left(\frac{\left(Y^{T} \cdot A^{-1} \cdot E\right)^{2}}{E^{T} \cdot A^{-1} \cdot E}-Y^{T} \cdot A^{-1} \cdot Y\right)
$$

is maximal likelihood estimation (MLE) of parameter $\beta$. 
Remark. Note that the degree $\delta$ is a perfect parameter of GRF as well, which can be estimated using the observation data. The least square estimate $\hat{\delta}$ is estimated by the univariate minimization of variance parameter MLE (6):

$$
\hat{\delta}=\arg \min _{0 \leq \delta \leq 1} \frac{1}{K} \cdot\left(\frac{\left(Y^{T} \cdot A^{-1} \cdot E\right)^{2}}{E^{T} \cdot A^{-1} \cdot E}-Y^{T} \cdot A^{-1} \cdot Y\right) .
$$

The properties of this estimate are explored by computer simulation in section 5 .

Note, that engineering processes are often observed only partially as in a case considered above. Surrogate modelling is a way to investigate the unobserved part of such processes (Carpio et al., 2017). Thus, the developed model (1), (2) presents the surrogate model of experimental data, because of its accurate prediction (even with a relative few points for fitting) and statistical interpretation that allows one to build an estimate of the potential error in a managerially efficient way.

\section{Optimal planning of extremal experiments}

In creating functional products and processes engineers are using the iterative experimental design process. Resource allocation exploring sets of possible alternatives requires to perform the experimental design process in an optimal way. Assume, the set of experiments $\hat{X}=\left(x_{N+1}, x_{N+2}, \ldots, x_{K}\right)$ should be planned at which the values of responsthe $e$ function $\hat{Y}=\left(y_{N+1}, y_{N+2}, \ldots, y_{K}\right)$ are predicted using the method given above. The efficiency of experimental the eries is evaluated by introducing the utility function

$$
H\left(\hat{Y}, y_{\max }\right)=\max \left(\max \left(y_{N+1}, . ., y_{K}\right)-y_{\max }, 0\right)
$$

where $y_{\max }=\max (Y)$.

By using expressions (4)-(6), the multi-dimensional normal distribution density function is written down as follows:

$$
\begin{gathered}
p(\hat{Y}, y(\hat{X}), \beta(\hat{X}))= \\
(2 \pi)^{-\frac{L}{2}} \cdot \sqrt{|\beta(\hat{X})|} \cdot \exp \left(-\frac{1}{2} \cdot(\hat{Y}-y(\hat{X}))^{T} \cdot \beta^{-1}(\hat{X}) \cdot(\hat{Y}-y(\hat{X}))\right),
\end{gathered}
$$

which describes the conditional distribution of predicted response function values in the subset $\hat{X}$.

Using (8), (9), one can write the expectation of the utility function as follows:

$$
U(\hat{X})=\int_{\max \left(y_{N+1}, . ., y_{K}\right) \geq y_{\max }}\left(\max \left(y_{N+1}, . ., y_{K}\right)-y_{\max }\right) p(Y, y(\hat{X}), \beta(\hat{X})) d Y .
$$

A series of new experiments should be planned to maximize the expected increment of the objective response function. Thus, the plan for an optimal experiment series is a solution to the equation:

$$
\begin{aligned}
& \hat{X}_{\text {opt }}=\arg \max _{X} \int_{\max \left(y_{N+1}, . ., y_{K}\right) \geq y_{\max }}\left(\max \left(y_{N+1}, . ., y_{K}\right)-y_{\text {max }}\right) . \\
& \ln (p(Y, y(X), \beta(X))) p\left(Y, y\left(\hat{X}_{o p t}\right), \beta\left(\hat{X}_{o p t}\right)\right) d Y \text {. }
\end{aligned}
$$

The gradient of this function with respect to the set of planned series is as follows:

$$
\begin{aligned}
& \frac{\partial U(\hat{X})}{\partial \hat{X}}=\int_{\max \left(y_{N+1}, . ., y_{K}\right) \geq y_{\max }}\left(\max \left(y_{N+1}, . ., y_{K}\right)-y_{\text {max }}\right) \cdot \frac{\partial \ln (p(Y, y(\hat{X}), \beta(\hat{X})))}{\partial \hat{X}} p(Y, y(\hat{X}), \beta(\hat{X})) \cdot d Y= \\
& E\left(\left(\max \left(y_{N+1}, . ., y_{K}\right)-y_{\max }\right) \cdot \frac{\partial \ln (p(Y, y(\hat{X}), \beta(\hat{X})))}{\partial \hat{X}}\right) .
\end{aligned}
$$

After some manipulations, one can make sure that the solution of (11) is the same as the solution to the minimization problem of the following objective function: 


$$
\begin{aligned}
& F(\hat{X})=\frac{1}{2}\left(\ln (|\beta(\hat{X})|) \cdot E\left(H\left(\hat{\mathrm{Y}}, y_{\max }\right)\right)+\operatorname{tr}\left(E\left(H\left(\hat{\mathrm{Y}}, y_{\max }\right) \cdot \hat{\mathrm{Y}} \cdot \hat{\mathrm{Y}}^{T}\right) \cdot \beta^{-1}(\hat{X})\right)+\right. \\
& \left.y(\hat{X})^{T} \cdot \beta^{-1}(\hat{X}) \cdot y(\hat{X}) \cdot E\left(H\left(, y_{\max }\right)\right)\right)-E\left(H\left(, y_{\max }\right) \cdot\right)^{T} \cdot \beta^{-1}(\hat{X}) \cdot y(\hat{X}) .
\end{aligned}
$$

Design of a series of experiments according to (13) is related to the calculation of complex multivariate integrals. The problem of (13) maximization is solved in an iterative way by the Monte Carlo method and the Sampling Average Approximation method (Shapiro (2013)). Let $M$ standard $(K-N)$ dimensional Gaussian vectors $v^{j}$ be simulated and fixed, $1 \leq j \leq M$. Assume $k$ iterations of the method to be performed and some subset $\hat{X}_{i}$ be obtained. Then the sample of response function values in the set $\hat{X}_{i}$ is computed in the following way:

$$
y^{j}=y\left(\hat{X}_{i}\right)+v^{j T} \cdot b\left(\hat{X}_{i}\right)
$$

where $b\left(\hat{X}_{i}\right) \cdot b\left(\hat{X}_{i}\right)^{T}=\beta\left(\hat{X}_{i}\right)$. One can then denote the Monte Carlo estimates as follows:

$$
\begin{gathered}
E\left(H\left(\hat{Y}, y_{\max }\right)\right) \approx \frac{1}{M} \cdot \sum_{j=1}^{M} H\left(y^{j}, y_{\max }\right) ; \\
E\left(H\left(\hat{Y}, y_{\max }\right) \cdot \hat{Y}\right) \approx \frac{1}{M} \cdot \sum_{j=1}^{M} H\left(y^{j}, y_{\max }\right) \cdot y^{j} ; \\
E\left(H\left(\hat{Y}, y_{\max }\right) \cdot \hat{Y} \cdot \hat{Y}^{T}\right)=\frac{1}{M} \cdot \sum_{j=1}^{M} H\left(y^{j}, y_{\max }\right) \cdot y^{j} \cdot y^{j T} .
\end{gathered}
$$

The estimates are inserted into expression (14), that is maximized by $\hat{X}$ for fixed estimates (15)-(17). Denote this intermediate optimization result as $\hat{X}_{i+1}$. Now, a new sample (14) is recalculated using $\hat{X}_{i+1}$ for computing conditional expectation (4), covariances (5), while the maximization of (13) is repeated. The process is stopped when the difference of results of two consecutive steps become negligible. The result of the last optimization presents us a $\mathrm{pl}$ with withan of the new experiment series.

Designed Bayesian plans distinguish with flexibility because they enable us to avoid of multi-factor experiments, usually applied in traditional engineering design. The key to using developed model for experimental optimization lies in balancing the need to exploit the approximating surface by sampling where it is minimized, with the need to improve the approximation by sampling where prediction error may be high.

\section{Computer modelling}

The behaviour of the developed model was investigated through a benchmark test function and an engineering optimization problem: optimal design of wastewater filter. The algorithm developed has been explored by computer simulation using the test function

$$
f(x, y)=\sqrt{(x-5)^{2}+(y-5)^{2}}, 0 \leq x \leq 10,0 \leq y \leq 10 .
$$

Developed data model depends on two parameters: variance $\beta^{2}$ and degree $\delta$, which can be estimated by maximal likelihood method, using (6), (7). Properties of these parametres have been explored by Monte Carlo method. The computer simulation experiment has been performed generating $N=200$ samples of $K=20$ randomly simulated points for test function in its domain. The histogram of vathe riance parameter $\beta^{2}$ is depicted in Figure 1, meathe $n$ of variance, and standard deviation are as follows: $\beta_{\text {Average }}^{2}=0.1924, \beta^{2}{ }_{\text {st.dev }}=0.024$. The histogram, of parameter $\delta$ is depicted in Figure 2, mean, and standard deviation are as follows: $\delta_{\text {Average }}=0.7537, \delta_{\text {st.dev }}=0.0093$. Maximal likelihood estimation of data model parameters (e.g. see Figure 1 and Figure 2). Thus, the explored benchmark showed that parameter of fraction $\delta$ is perfe the ct parameter of the created a model. 


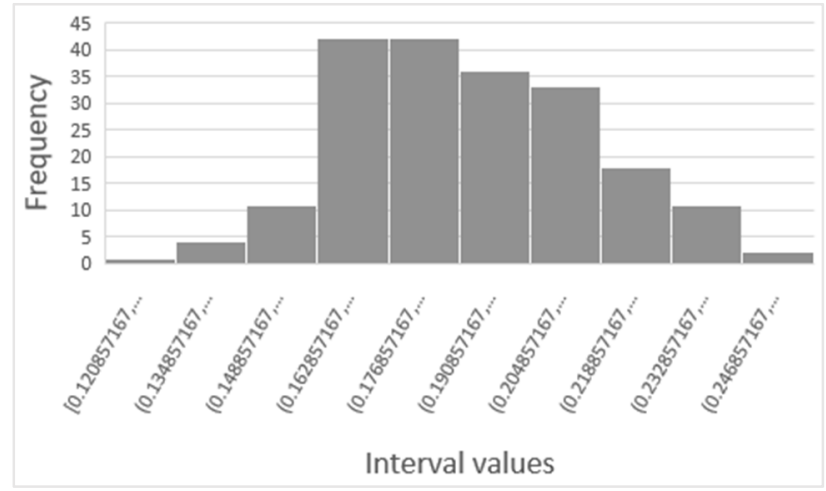

Figure 1. Variance parameter, $\beta$

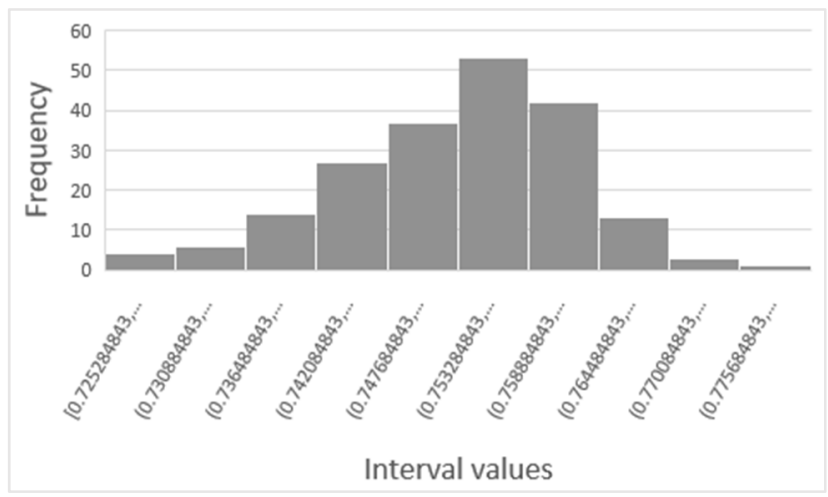

Figure 2. Optimal Euclidean fraction degree, $\delta$

Further, the algorithm for experiment series planning has been explored by the Monte Carlo method. The set from $N=10$ has been uniformly randomly simulated in the feasibility set and respective values of the test function have been computed. The minimal value of the test function was established: $y_{\min }=1.5300419$. Next, the initial values of the planned series have been chosen randomly:

$$
x_{1}=\left(\begin{array}{cc}
5.7588904 & 5.5526906 \\
6.23848 & 5.0890846
\end{array}\right),
$$

the value of the function (8) was 0.1977166 , and after optimization, this value achieved 0.1239016 . The solution of this optimization was:

$$
x_{2}=\left(\begin{array}{ll}
4.6325385 & 5.3802934 \\
4.1278819 & 4.9403434
\end{array}\right),
$$

the value of function (8) 0.201207 and after optimization 0.1995037 . The new solution of the latter task

$$
x_{3}=\left(\begin{array}{ll}
4.7138052 & 5.4645915 \\
4.1690978 & 5.0429421
\end{array}\right)
$$

has been minimized, the function value at these points was 0.2056391 , which after the optimization changed insignificantly and therefore it was taken as a new series for experiment planning. The values of the test function at the points of the proposed series $x_{3}$ were 0.5456672 and 0.8320111 , which illustrates the increment of the response function in the planned series. Thus, computer experiment confirms the applicability of the developed approach for experimental optimization of response surface in engineering design. 


\section{Modelling of effectiveness of surface wastewater treatment filter filler}

The created method is applied for solving of engineering problem: optimal design of wastewater filters. Let $x=\left(x_{1}, x_{2}, x_{3}, x_{4}\right)^{T}$ be filler ratios vector, where $x_{1}-$ quartz sand (QS), $x_{2}$ - shredded autoclaved aerated concrete (SHAAC), $x_{3}$-stone wool (SW), $x_{4}$ - biochar (BC). Denote the vector of results of filter characteristics measurement: $Y_{i}^{j}, 1 \leq i \leq m, 1 \leq j \leq K$, where $K=4$ - number of experiments, and $m$-number of filter characteristics. Assume these filter characteristics be describing capability to treat different wastes. By the example, these filters with different fillers are designed for treatment of main pollutants of the surface wastewater by total carbon (TC).

The experiments, where performed with four filters filled by following fillers:

1 - Quartz sand (100\%);

2 - Shredded autoclaved aerated concrete $(66.7 \%)$ and stone wool $(33.3 \%)$;

3 - Shredded autoclaved aerated concrete $(33.3 \%)$ and biochar $(66.7 \%)$;

4 - Shredded autoclaved aerated concrete (33.3\%), biochar (33.3\%) and stone wool (33.3\%).

Thus, the experiment matrix of filler proportions is as follows:

$$
X=\left[\begin{array}{cccc}
1 & 0 & 0 & 0 \\
0 & 0.667 & 0 & 0.333 \\
0 & 0 & 0.333 & 0.667 \\
0 & 0.333 & 0.333 & 0.333
\end{array}\right] .
$$

The filtration characteristics measurement of element $T C$, at this experiment matrix, were:

\begin{tabular}{|c|c|}
\hline Filter & $T C$ \\
\hline 1 & 27.3 \\
\hline 2 & 38.3 \\
\hline 3 & 50.7 \\
\hline 4 & 43.2 \\
\hline
\end{tabular}

The new plan for proportions for filter fillers by the developed method has been created:

$$
\hat{X}=\left[\begin{array}{cccc}
0 & 1 & 0 & 0 \\
0 & 0 & 0 & 1 \\
0.333 & 0 & 0 & 0.667 \\
0 & 0 & 0.16 & 0.84
\end{array}\right]
$$

Performance of series from 4 experimental filters according to the plan $\hat{X}$ should ensure the new construction of filter with increased efficiency of the filter.

\section{Conclusions}

Creation of functional products and processes using the experimental design often deals with problems of engineering economy, because of the necessity to carry out parts of the design process seeking economically and managerially efficient resource allocation. This paper deals with the Bayesian experimental design method, based on the interpretation of observations data by random Gaussian fields, obtained during the experiments. The findings obtained allows us to include the predictable information about the engineering design variables into the model in order to identify and estimate the uncertainties of observations. Using this approach, the novel method is created to plan a series of extremal experiments by maximizing the expected increment on the response surface. The created method is investigated by computer simulation for solving test examples and is applied in the optimal design of wastewater filters as well. The developed approach enables us to design the experimental series inflexible way, decreasing the number of required experiments and avoiding of rather expensive traditional methods, such as factorial experiments, steepest descent, etc. Although the creation of the plans of the experiment series can require a large amount of computer time related with the application of Monte Carlo procedure in order to ensure the optimality of created plans, this limitation can be avoided using distributed computing tools, besides, this amount of computer time often meets requirements in real design practice. Hence, theoretical investigation, solving of the benchmark example and application to real engineering design, enable us to conclude that the created method helps engineers to seek solutions to experimental problems, considering the economic viability of each potential solution along with the technical aspects. 


\section{References}

Bijan, V., \& Gorjidooz, J. (2016). Engineering economics for aviation and aerospace. Taylor \& Francis.

Blondet, G., Le Duigou, J., \& Boudaoud, N. (2019). A knowledge-based system for numerical design of experiments processes in mechanical engineering. Expert Systems with Applications, 122, 289-302. https://doi.org/10.1016/j.eswa.2019.01.013

Box, G. E. P., \& Draper, N. R. (2007). Response Surfaces, Mixtures, and Ridge Analyses. Hoboken, NJ, USA: John Wiley \& Sons, Inc. https://doi.org/10.1002/0470072768

Carpio, R., Giordano, R. C., \& Secchi, A. (2017). Enhanced surrogate assisted global optimization algorithm based on maximizing probability of improvement. Computer Aided Chemical Engineering, 40, 2065-2070. https://doi.org/10.1016/B978-0-444-63965-3.50346-9

El-Gamal, M.A., \& Palfrey, T. R. (1996). Economical experiments: Bayesian efficient experimental design. International Journal of Game Theory, 25(4), 495-517. https://doi.org/10.1007/BF01803953

Jones, D. R. (2001). A taxonomy of global optimization methods based on response surfaces. Journal of Global Optimization, 21, 345-383. https://doi.org/10.1023/A:1012771025575

Nooshin, A., \& Hamid, Z. (2017). Optimization of organosolv pretreatment of rice straw for enhanced biohydrogen production using Enterobacter aerogenes. Bioresource Technology, 227, 335-344. https://doi.org/10.1016/j.biortech.2016.12.073

Maged, A., Haridy, S., Shamsuzzaman, M., Alsyouf, I., \& Zaied, R. (2018). Statistical monitoring and optimization of electrochemical machining using shewhart charts and response surface methodology. International Journal of Engineering Materials and Manufacture, 3(2), 68-77. https://doi.org/10.26776/ijemm.03.02.2018.01

Morris, W. T. (2013). Engineering economy: the analysis of management decisions.

Pozniak, N., \& Sakalauskas, L. (2017). Fractional euclidean distance matrices extrapolator for scattered data. Jaunuju mokslininku darbai, 2(47), 56-61. https://doi.org/10.21277/jmd.v47i2.156

Pozniak, N., \& Sakalauskas, L. (2019). Kriging and extrapolation with fractional Euclidean distance matrices. Informatica, 30(2), $1-23$.

Shapiro, A. (2013). Sample Average Approximation, 1350-1355. https://doi.org/10.1007/978-1-4419-1153-7_1154

Weinberger, K. Q., Sha, F., \& Saul, L. K. (2004). Learning a kernel matrix for nonlinear dimensionality reduction. Proceedings of the twenty-first international conference on Machine learning (ICML '04), 106-113.

https://doi.org/10.1145/1015330.1015345 